\title{
Atrial fibrosis in atrial fibrillation pre-ablation assessed by CMR: impact in atrial size and function?
}

\author{
Ana G Almeida*, Joao Sousa, Luis Carpinteiro, Joao S Marques, Nuno Cortez-Dias, Claudio David, Claudia Jorge, \\ Doroteia Silva, Mario G Lopes, Antonio N Diogo
}

From 2011 SCMR/Euro CMR Joint Scientific Sessions

Nice, France. 3-6 February 2011

\section{Introduction}

Atrial fibrillation (AF) is associated with different amounts of diffuse fibrosis, which has impact in the therapy response. CMR is able to assess myocardial fibrosis using late gadolinium enhancement (LGE) and chamber function.

\section{Purpose}

The aim of this study was to assess, in patients with AF if the presence and extension of atrial fibrosis was related with atrial size and function.

\section{Methods}

47 (42 male, $42 \pm 8$ years-old) consecutive patients (pts) with paroxysmal AF and indication to ablation were included. Exclusion criteria for CMR: non-sinus rhythm and general contra-indications. Before ablation all underwent CMR for left atrial angiography for CARTO mapping. Using CMR, the following variables were assessed: left ventricle (LV) and left atrium (LA) volumes and ejection fraction (SSFP, short-axis), LV LGE was assessed as presence and amount (LGE mass/LV mass). LA LGE location was assigned to 3 atrial walls: anterior, posterior and septal. The extension was classified in 3 degrees, according to the number of atrial walls with LGE: $1=$ one wall, $2=$ two walls; $3=$ three walls. For CMR analysis dedicated software was used (Philips View Forum Release 6.1).

\section{Results}

All studies yielded good image quality. LV end-diastolic volume was $68 \pm 5 \mathrm{ml} / \mathrm{m} 2$ and normal in all; LV ejection fraction was $58 \pm 4 \%(48-67)$, normal in all (in 2 borderline);
11 pts showed minimal LV LGE involving one or two segments with $0.02 \%$ of fibrosis mass/LV mass; mean LA volume was $115 \pm 21 \mathrm{ml}$ (increased in $45 \%$ o patients); mean LA ejection fraction was $45 \pm 11 \%$ (reduced in $65 \%$ ). Type 1 LGE was found in 17 pts, type 2 in 22 and type 3 in 8 pts. Comparing the groups with the 3 LGE types, pts with type 3 LGE had larger LA volumes and lower ejection fraction in comparison with type 1 LGE $p t s(p=0.004$ and $p=0.001$ respectively). No difference was found between other groups. No relationship was found with LV volumes, ejection fraction and amount of fibrosis.

\section{Conclusion}

In pts with paroxysmal AF pre-ablation, atrial fibrosis as assessed semi-quantitatively by CMR was associated to larger left atrial volume and lower ejection fraction, which are determinant substrates for the outcome.

Published: 2 February 2011

doi:10.1186/1532-429X-13-S1-P259

Cite this article as: Almeida et al:: Atrial fibrosis in atrial fibrillation preablation assessed by CMR: impact in atrial size and function? Journal of Cardiovascular Magnetic Resonance 2011 13(Suppl 1):P259.

University Hospital Santa Maria, Lisbon, Portugal

(c) 2011 Almeida et al; licensee BioMed Central Ltd. This is an open access article distributed under the terms of the Creative Commons 